\title{
Hippocampal volume and functional connectivity changes during the female menstrual cycle ${ }^{2}$
}

\author{
Nina Lisofsky ${ }^{\mathrm{a}, *}$, Johan Mårtensson ${ }^{\mathrm{b}}$, Anne Eckert ${ }^{\mathrm{c}}$, Ulman Lindenberger ${ }^{\mathrm{a}}$, Jürgen Gallinat ${ }^{\mathrm{d}}$, Simone Kühn ${ }^{\mathrm{a}, \mathrm{d}}$ \\ a Max Planck Institute for Human Development, Berlin, Germany \\ b Lund University, Lund, Sweden \\ c Psychiatric University Clinics, University of Basel, Basel, Switzerland \\ d Department of Psychiatry and Psychotherapy, University Medical Center Hamburg-Eppendorf, Germany
}

\section{A R T I C L E I N F O}

\section{Article history:}

Received 19 February 2015

Accepted 3 June 2015

Available online 6 June 2015

\section{Keywords:}

Hormones

Hippocampus

Structural MRI

Resting state connectivity

\begin{abstract}
A B S T R A C T
Hippocampal volume has been shown to be sensitive to variations in estrogen and progesterone levels across rodents' estrous cycle. However, little is known about the covariation of hormone levels and brain structure in the course of the human menstrual cycle. Here, we examine this covariation with a multi-method approach that includes several brain imaging methods and hormonal assessments. We acquired structural and functional scans from 21 naturally cycling women on four time points during their cycles (early follicular phase, late follicular phase, ovulation and luteal phase). Hormone blood concentrations and cognitive performance in different domains were assessed on each of the measurement occasions. Structural MRI images were processed by means of whole-brain voxel-based morphometry and FreeSurfer. With either method, bilateral increases in hippocampal volume were found in the late follicular phase relative to the early follicular phase. The gray matter probability in regions of hippocampal volume increase was associated with lower mean diffusivity in the same region. In addition, we observed higher functional connectivity between the hippocampi and the bilateral superior parietal lobe in the late follicular phase. We did not find any reliable cycle-related performance variations on the cognitive tasks. The present results show that hormonal fluctuations covary with hippocampal structure and function in the course of the human menstrual cycle.
\end{abstract}

(c) 2015 Elsevier Inc. All rights reserved.

\section{Introduction}

The variations of female sex hormones over the menstrual cycle in mammals are connected to neural changes, including structural as well as functional transformations in different brain regions (e.g., Czoty et al., 2009; Desmond and Levy, 1997; Olmos et al., 1989; Qiu et al., 2013; Woolley, 1998; Woolley and McEwen, 1993). Estrogen and progesterone, the two main ovarian steroids, have received most attention in this research field. A multitude of animal studies has revealed detailed insights into hormone-related structural brain alterations. However, only a few studies investigated this topic in humans, and many of the previous studies lack adequate hormonal assessments or hypothesis-free whole-brain neuroimaging methods.

Basic knowledge about the association between the estrous cycle of female rats and neuroplasticity comes from a number of studies conducted by Woolley and colleagues. Naturally occurring variations in

it Nina Lisofsky has been a pre-doctoral fellow of the International Max Planck Research School on the Life Course (LIFE, www.imprs-life.mpg.de; participating institutions: MPI for Human Development, Freie Universität Berlin, Humboldt-Universität zu Berlin, University of Michigan, University of Virginia, University of Zurich).

* Corresponding author at: Max-Planck-Institute for Human Development, Lentzeallee 94, 14195 Berlin, Germany.

E-mail address: lisofsky@mpib-berlin.mpg.de (N. Lisofsky). estrogen and progesterone have been shown to covary with spine density in the cornu ammonis 1 (CA1) hippocampal pyramidal cells (Woolley and McEwen, 1990). Increasing estrogen levels were accompanied by increasing spine density. In further experiments it was documented that not only spine density in the hippocampus increases following rise of estrogen, but that new synapses are built and the sensitivity of the synapses (to NMDA receptor-mediated input) is increased (Woolley et al., 1996, 1997). Estrogen effects on the brain are altered by progesterone and might vary between species (Pawluski et al., 2009; Woolley and McEwen, 1993). Following up on these seminal studies, researchers explored estrogen-dependent variations in cognitive function. Because the hippocampus has been demonstrated to be one of the main hormone-sensitive targets in the brain, hippocampus dependent cognitive functions, such as spatial-memory tasks, were investigated. Results are mixed, showing differences in associations to endogenous and exogenous hormonal variation, dose-and durationspecific reactions, and interactions between different gonadal hormones on cognitive functions (e.g., Bimonte and Denenberg, 1999; Chesler and Juraska, 2000; Gibbs, 2000; Korol and Kolo, 2002; O'Neal et al., 1996; Stackman et al., 1997). Some studies find evidence for decreased spatial abilities (i.e., spatial learning and spatial recognition memory) in high-estrogen phases during the cycle (e.g., Galea and Kavaliers, 1995; Lacreuse et al., 2001). Other studies revealed that 
estrogen administration in ovariectomized rats results in better spatial performance (i.e., spatial working memory; Fader et al., 1998; O'Neal et al., 1996). Lastly, some researchers report stable spatial-memory performance across the estrous cycle (Stackman et al., 1997). Results are more convergent for spatial strategies used to solve navigation tasks: rats prefer allocentric (hippocampal-based) strategies when exposed to high estrogen levels and egocentric (striatal-based) strategies when exposed to low levels (Hussain et al., 2014; Korol et al., 2004; Qiu et al., 2013). The different degrees to which spatial memory tasks rely on these learning strategies make comparisons between studies difficult.

More recently, hormone-related variations in cognition and neural plasticity in human females have come under empirical investigation. Compared to the five-day estrous cycle in rats, the human menstrual cycle takes about 28 days, and can be subdivided into four phases: first, the early follicular phase (EFP) during menses, where the steroid concentrations are low; second, the late follicular phase (LFP), where estrogen is at its highest levels and progesterone, luteinizing hormone (LH) and follicle-stimulating hormone (FSH) are low; third, the ovulation, with LH and FSH at their peaks; fourth, the luteal phase, in which estrogen and progesterone are both at high levels. Most studies so far compared the luteal with the early follicular phase to arrive at a high estrogen/progesterone vs low estrogen/progesterone contrast. Looking at cognitive performance, a number of studies have reported lower spatial (e.g., mental rotation) performance during cycle phases with high estrogen/progesterone levels (e.g., Hampson, 1990; Hausmann et al., 2000; Silverman and Phillips, 1993). In contrast, other studies show no overall influence of varying estrogen/progesterone levels on those abilities (e.g., Epting and Overman, 1998; Rosenberg and Park, 2002). In opposition to spatial abilities, verbal memory performance was shown to improve with high estrogen levels (Rosenberg and Park, 2002), but again, not all studies have found effects of menstrual cycle phases in this domain (Mihalj et al., 2014). The difficulty to separate memory and spatial skills in cognitive tasks hampers the comparison of results across studies.

Only few studies so far have looked at hormone-related structural plasticity in the female brain. Hagemann et al. (2011) investigated short-term whole-brain volume changes in total gray matter (GM) across the menstrual cycle. They found a GM volume peak along with a reduction of cerebrospinal fluid at time of ovulation. Individual differences in the magnitude of these volume changes were not reliably related to changes in estrogen or progesterone hormone levels between the respective phases. Ossewaarde et al. (2011) restricted their analysis to changes in the amygdala. Measuring the participants on two different time points during the cycle (follicular and luteal phase), they found an increase in the dorsal part of the left amygdala in the luteal phase. In a recent voxel-based morphometry (VBM) study, De Bondt et al. (2013) found structural changes in a number of frontal and temporal regions when comparing the follicular and luteal phase of 15 young, naturally cycling women. Larger GM volumes during the follicular phase were found inter alia in the anterior cingulate cortex (ACC) and fusiform gyrus. In addition, a negative correlation between estrogen and GM volume in the ACC was found during the luteal phase. The authors discuss these neural changes in relation emotional processing in women across the menstrual cycle. The results of two other studies applying VBM (Pletzer et al., 2010; Protopopescu et al., 2008) are in line with findings from the animal literature. Both studies show evidence for structural changes in the hippocampal formation during the menstrual cycle. Pletzer et al. (2010) found an increase in a right fusiform/ parahippocampal gyrus region of interest (ROI) during the early follicular (low hormonal) phase, compared to the mid-luteal (high hormonal) phase. In contrast Protopopescu et al. (2008) found a right hippocampal GM increase during the peak estrogen late follicular phase, compared to the late luteal (low hormonal) phase. Furthermore, Protopopescu et al. (2008) found that verbal memory was increased during the peakestrogen phase, pointing to a connection between cognitive and brain structural changes. In both studies, participants were measured at two time points during their cycle, but the exact cycle phases measured differ between the studies, rendering direct comparisons difficult. In addition, neither of the two studies assessed hormonal levels, which further weakens the conclusions that can be drawn from these studies. It remains unclear, whether the knowledge about cycle-related hippocampal plasticity can be transferred from animal studies to humans. The animal pattern predicts that the hippocampal volume follows the estrogen increase from early to late follicular phase and stays high until the luteal phase, where it starts decreases again due to elevated progesterone levels. This pattern would suggest more strongly that the same mechanisms underlying hormone-related hippocampal plasticity in animals come in consideration in humans.

To summarize, the human structural magnetic resonance imaging (MRI) literature provides some initial evidence that structural changes over the menstrual cycle are present in women. Almost all of the available studies are restricted to two time points only, and hence give a very impoverished picture of the hormonal changes during the menstrual cycle and their potential relations to the structure and function of the brain. In addition, actual hormone levels are often not measured. Finally, differences in the timing of measurements in relation to the menstrual cycle additionally hinder comparisons across studies. Reliable withinsubject differences in brain structure and function of women during the menstrual cycle would have important implications for neuroimaging research. A proportion of observed between-subject variance might be explained by within-person fluctuations and this cycle-related variability could distort cross-sectional and longitudinal neuroimaging findings.

With the present study we aimed at investigating structural and functional brain changes over the female menstrual cycle by measuring young women at four different occasions according to the four cycle phases. Following up on the studies by Pletzer et al. (2010) and Protopopescu et al. (2008), we expected that the hippocampus is especially sensitive to hormonal variation. To test this assumption most conservatively, we applied whole-brain voxel based comparisons that are uninfluenced by our a priori hypotheses. Based on animal results (Woolley, 1998), we hypothesized hippocampal structure and connectivity to increase from low to high estrogen phases. We mainly expected hippocampal differences when contrasting early and late follicular phase. This contrast compares a high and low estrogen phase, while being relatively unaffected by progesterone levels. In the luteal phase, effects of progesterone might attenuate the effects of estrogen. Based on this main phase contrast, we wanted to investigate the pattern of hippocampal variation across the whole menstrual cycle. Corresponding to estrogen levels, we expected to find increased volume in late follicular, ovulatory and luteal phase, but volume at ovulation and luteal phase might be lower compared to late follicular phase, indicating oppositional effects rising progesterone levels. We also included cognitive variables to explore whether cognitive performance would vary across cycle phases, and covary with changes in estrogen levels.

\section{Methods}

\subsection{Participants}

Twenty-five right-handed, healthy women were included in the initial study sample after written informed consent. The study was conducted according to the Declaration of Helsinki, with approval of the German Psychological Society ethics committee. All women reported regular menstrual cycles (mean 28 days, range 26-30 days). We recruited women who did not use hormonal contraceptives during the three months preceding the study. All participants reported no history of neurological or psychiatric conditions or of drug/alcohol abuse. Four women participated in only two of the four measurement occasions and were excluded from the analyses, because information on the 
course of their cycle was missing. The final sample consisted of 21 women (mean age: $26.8 \pm 2.45$ (SD), range 22-31).

\subsection{Design and procedure}

The women were asked to monitor their menstrual cycle at least one cycle before the measurement phase started. During this time the onset of the menses, time of estimated ovulation (based on urinary LH-tests) and the length of the cycle were noted down. This information was used to schedule the measurement times individually for each woman.

The women were tested four times across the cycle (Table 1). One measurement took place during the early follicular phase (EFP), that is, between the first and the third day after onset of menses. Another measurement was scheduled during the late follicular phase (LFP), two to three days before expected ovulation. A third measurement was taken around the time of ovulation (OVP) as verified by means of peak urine LH tests, in the middle of the cycle at day 13 to 17 after onset of menses. The women measured their urine LH concentration every morning during mid-cycle and informed the experimenters about detected increases so that a corresponding measurement occasion could be scheduled. Resulting from different cycle-lengths, women started to assess the LH concentration between days 9 and 13 of their cycle. A fourth measurement was taken during the mid-luteal phase (MLP), that is, at day 20 to 24 after onset of menses.

To avoid systematic training effects in the cognitive tasks, the cycle phase at which women were scanned first was randomly assigned to the participants. The measurement occasions following the first occasion were scheduled following the cycle, so that a women starting in the OVP, for instance, would have her second, third, and fourth measurement occasions during MLP, EFP, and LFP, respectively. Due to this randomization procedure, some women were measured in parts of two consecutive cycles. Each testing session took place between 8 am and $1 \mathrm{pm}$. The four sessions of each individual participant were scheduled within a window of $2 \mathrm{~h}$ on each of the four days, to hold diurnal effects on hormone concentration constant at the individual level. The scanning procedure lasted for about an hour. Afterwards a blood sample was taken, and the women completed the cognitive test battery.

\subsection{Behavior}

A selection of computerized tests from the Cogito study (Schmiedek et al., 2010) was administered to assess a broad range of cognitive abilities. On each of the four measurement occasions, we applied a different version of the tasks, containing different items. We included the following tasks: Two tasks assessing perceptual speed (numerical and figural comparison), in which participants had to compare two five-numberstrings or two three-dimensional figures presented on the left and right part of the screen. Two tasks assessing working memory (memory updating, 2-back spatial). In the memory updating task, participants had to apply an order of eight mathematical operations, each simultaneously on four memorized digits presented initially on the screen. In the spatial n-back tasks, participants had to follow a dot appearing at different positions in a 4 by 4 grid and indicate whenever the dot appeared at the same position as the item two steps earlier. Three tasks assessing episodic memory (word-list recall, number-noun pairs, object position memory), in which participants had to memorize a list of 26

Table 1

Measurement occasions and expected hormonal levels.

\begin{tabular}{llllll}
\hline Phase & Time & E & P & LH & FSH \\
\hline Early follicular phase (EFP) & Days 1-3 after onset of menses & L & L & L & H \\
Late follicular phase (LFP) & Days 10-13 after onset of menses & H & L & L & L \\
Ovulation (OVP) & Days 13-17 after onset of menses & H & L & H & H \\
Mid-luteal phase (MLP) & Days 20-24 after onset of menses & H & H & L & L \\
\hline
\end{tabular}

$\mathrm{E}=$ estrogen; $\mathrm{P}=$ progesterone, $\mathrm{LH}=$ luteinizing hormone; $\mathrm{FSH}=$ follicle stimulating hormone; $\mathrm{L}=$ low; $\mathrm{H}=$ high. words, a combination of a two-digit number and a noun (the noun was presented and the number had to be recalled) and the position of presented objects on a grid. With exception for the object-location task, correct responses (or number of recalled words/nouns) were used as the main outcome measure for each task. In the object location task, the distance of the placed object to the correct object on the grid was used as the outcome measure. Additionally, the GuilfordZimmerman spatial orientation test (Guilford and Zimmerman, 1948), a paper-pencil measure of the ability to process spatial relations, was administered at each measurement occasion. The long version of that task was divided into four parts, each containing 15 items, for which the participants were given 4 min of time. Performance was analyzed in a multilevel models predicting the cognitive outcome measure by cycle phase and including a regressor for the order of test sessions to control for retest effects. A $P$-value of $P>0.006$ was determined by Bonferroni correction to control for multiple testing.

\subsection{Hormonal assessment}

To verify ovulation, the women assessed their LH urine concentration with a commercially available ovulation predictor kit (One Step LH Urine Ovulation Test (Strip), manufactured by: AIDE DiagnosticaCOo. Ltd.). Blood samples were taken on each of the measurement occasions. All of the blood was collected into commercial available $10 \mathrm{~mL}$ EDTAtubes. Plasma was obtained by centrifugation of blood samples by $2000 \mathrm{~g}$ at $8{ }^{\circ} \mathrm{C}$. The hormonal levels were assessed by simultaneously measuring analytes using magnetic bead technology and CCD imaging. Sample preparation and assays were performed according to the manufacturer's protocol (Millipore, Switzerland). Estrogen and progesterone were quantitatively determined using the Milliplex MAP Steroid Hormone Magnetic Bead Panel (Millipore, Switzerland). Extracted (using Waters manifold system with the Oasis HLB 96-well plate, $60 \mathrm{mg}$ sorbent per well, $60 \mu \mathrm{m}$ particle size; Waters AG, Switzerland) plasma samples were diluted 1:5 to be within the dynamic range of the assay. Regarding progesterone concentrations $>1 \mathrm{ng} / \mathrm{mL}$, a final sample dilution of 1:20 and reanalysis for just that particular analyte (progesterone at MLP) was required. LH and FSH (undiluted plasma samples) were quantified using Milliplex MAP Human Pituitary Magnetic Bead Panel 1 (Millipore, Switzerland). Samples ( $25 \mu \mathrm{L}$ ) were measured in the Luminex Multiplexing Instrument MAGPIX System from Millipore, Switzerland. Serum BDNF levels were assessed with an enzyme-linked immunoabsorbent assay (ELISA) kit (Promega BDNF Emax ${ }^{\circledR}$, Madison, Wis.). Detection of total soluble BDNF was carried out following the manufacturers' protocol (serum samples were diluted 1:100). The hormonal levels were compared by paired t-test applying an alpha level of .05. Statistical outliers were excluded before analysis (outliers were defined as values exceeding or falling below the 75 th/25th percentile by more than 1.5 times the interquartile range).

\subsection{MRI data acquisition}

Magnetic resonance images were acquired using a $3 \mathrm{~T}$ Magnetom Tim Trio MRI scanner system (Siemens Medical Systems, Erlangen, Germany) and a 12-channel radiofrequency head coil. High-resolution anatomical images were collected using a T1-weighted 3D MPRAGE sequence $(\mathrm{TR}=2500 \mathrm{~ms}, \mathrm{TE}=4.77 \mathrm{~ms}, \mathrm{TI}=1100 \mathrm{~ms}$, acquisition matrix $=256 \times 256 \times 192$, sagittal FOV $=256 \mathrm{~mm}$, flip angle $=7^{\circ}$, voxel size $=1.0 \times 1.0 \times 1.0 \mathrm{~mm}^{3}$ ). Whole-brain functional images were acquired using a $2^{*}$-weighted echo-planar-imaging (EPI) sequence sensitive to BOLD contrast (TR $=2000 \mathrm{~ms}$, TE $=30 \mathrm{~ms}$, image matrix $=72 \times 72$, FOV $=216 \mathrm{~mm}$, flip angle $=80^{\circ}$, slice thickness $=2.0 \mathrm{~mm}$, distance factor $=20 \%$, voxel size $3.0 \times$ $3.0 \times 3.0 \mathrm{~mm}^{3}, 36$ axial slices). 150 image volumes aligned to AC-PC were acquired. During this resting state measurement subjects were instructed to keep their eyes closed and relax. Diffusion-weighted images 
were attained with a single-shot diffusion-weighted spin-echo-refocused echoplanar imaging sequence (TR $=8000 \mathrm{~ms}$; TE $=93 \mathrm{~ms} ; 128 \times 128$ matrix interpolated to $256 \times 256$; FOV $224 \mathrm{~mm} \times 224 \mathrm{~mm}$; slice thickness $2 \mathrm{~mm}$; $b$-value $1000 \mathrm{~s} / \mathrm{mm}^{2}$; 62 slices). Additionally, the water content of the tissue was assessed, using a quantitative $\mathrm{T}^{*}$ sequence $(\mathrm{TR}=1000 \mathrm{~ms}, \mathrm{TE}=4 \mathrm{~ms}$, readout bandwidth $=592 \mathrm{~Hz} /$ pixel, image matrix $=256 \times 256$, FOV $=200 \times 200 \mathrm{~mm}^{2}$, flip angle $=90^{\circ}$, slice thickness $=2.0 \mathrm{~mm}, 64$ points measured on the T2* relaxation curve with an equidistant spacing of $15 \mathrm{~ms}$; Neeb et al., 2008; Tonkova et al., 2012). In order to get precise T1-, total water content- and myelin water content maps, the protocol included 2 multi-echo gre-sequences followed by three short EPI sequences. The first gre-sequence was used to generate the uncorrected signal intensity extrapolated to zero echotime that was than corrected for imperfection in the receiver coil, B1 field inhomogeneity and signal saturation effects due to T1 differences. Additionally the signal was scaled due to temperature differences between reference probe and body. All correction terms are averaged over all voxels to obtain a quantitative water content map. Water content within the hippocampus was determined and compared for differences between the cycle phases using an alpha level of .05. More detailed information on the sequence and the analysis procedure can be found in the paper by Neeb et al. (2008).

\subsection{MRI data analysis}

\subsubsection{Structural data analysis}

The MPRAGE structural brain data were first normalized using the open source software Advanced Neuroimaging Tools (ANTs, http:// www.picsl.upenn.edu/ANTS/). In a first step, we created a template of all images of the participants in this study by means of the buildtemplateparrallel script (Avants et al., 2010). The algorithm builds an average shaped brain within the diffeomorphic space (see Klein et al., 2010). Afterwards, all images were normalized to this template, using the antsIntroduction script (Avants et al., 2011). For both steps, cross-correlational similarity metric was used and a diffeomorphic image registration using Greedy SyN was applied. Different evaluation studies suggest that ANTS-based normalization is the most stable and reliable registration method currently available (Avants et al., 2008; Klein et al., 2010). The anatomical images were preprocessed afterwards by means of the VBM8 toolbox (http://dbm. neuro.uni-jena.de/vbm/) with default parameters.

The VBM8 default longitudinal preprocessing involves intra-subject realignment, intra-subject bias correction, tissue classification and normalization. The baseline (or mean) of the realigned images of one subject is used as a reference for realignment and signal-inhomogeneity correction. The normalization parameters of the baseline image are applied to the segmented, bias-corrected images, which are then again realigned. Afterwards, images were smoothed with a full-width halfmaximum (FWHM) kernel of $4 \mathrm{~mm}$. Statistical analysis was carried out by means of a flexible factorial design, comparing GM volume between two cycle phases for all subjects. All analyses were based on whole-brain comparisons. The contrasts were thresholded with $P<0.0005$ and combined with a non-stationary smoothness correction and an additional cluster size threshold of $k>25$ voxels (Hayasaka and Nichols, 2004). The main interest was the LFP $>$ EFP contrast. Significant clusters in that contrast in or near the hippocampus were followed up in further analyses to investigate potential underlying effects driving volumetric change (DTI, water content, GM volume across all four phases). Secondly, we were also interested whether hippocampal differences can be found in whole-brain analyses in any other than the proposed contrast. However, other brain regions showing significant differences in the other contrasts were not the main focus of the present investigation and are reported as supplementary material. GM values for each participant in the statistically significant regions within or nearby the hippocampus were extracted by means of MarsBaR region of interest toolbox for SPM (http://marsbar.sourceforge.net/) and correlated with the diffusion-weighted data within the respective cycle phases. As a secondary exploratory analysis, a within-subject whole-brain regression was performed in VBM8, using the estrogen values from the blood samples across the four measurement occasions. The resulting spm-con-maps were included in a second-level one-sample t-test to assess trends in the correlations between subjects.

We additional inspected hippocampal volume across the four phases with a different measure to see whether the expected pattern emerges. The hippocampal volumes of all participants at each occasion were measured using the fully-automated FreeSurfer hippocampus segmentation, a method to obtain hippocampus volumes automatically, which has been shown to compare well with the gold standard of manual tracing (Morey et al., 2009). The raw images were preprocessed using the FreeSurfer longitudinal stream and a $P<0.05$ threshold was applied to the paired t-test of hippocampal volume segmentations between LFP and EFP.

\subsubsection{DTI data analysis}

The diffusion weighted images were analyzed using the FSL software package (http://fsl.fmrib.ox.ac.uk/fsl/fslwiki/). Mean diffusivity (MD) images were created by fitting a tensor model to the raw diffusion weighted images using FDT. Brain extraction was done using BET (Smith, 2002) before alignment into common space using FNIRT (Andersson et al., 2007a,b). From the resulting normalized MD image, the MD-values for each participant in the hippocampal ROI resulting from the SPM contrast LFP > EFP were extracted using FSLUTILS.

\subsubsection{Functional data analysis}

The first five EPI volumes were discarded to allow the magnetization to approach a dynamic equilibrium. Part of the data pre-processing, including slice timing, head motion correction (a least squares approach and a 6-parameter spatial transformation) and spatial normalization to the Montreal Neurological Institute (MNI) template (resampling voxel size of $3 \times 3 \times 3 \mathrm{~mm}^{3}$ ), were conducted using the SPM8 and data processing assistant for resting-state fMRI (DPARSF, Chao-Gan and Yu-Feng, 2010). A spatial filter of $4 \mathrm{~mm}$ FWHM was used. Participants showing head motion above $3 \mathrm{~mm}$ of maximal translation (in any direction of $\mathrm{x}, \mathrm{y}$ or $\mathrm{z}$ ) and $1.0^{\circ}$ of maximal rotation throughout the course of scanning would have been excluded. None of the participants showed head movements exceeding these boundaries. After preprocessing, linear trends were removed. Then the fMRI data were temporally band-pass filtered $(0.01-0.08 \mathrm{~Hz})$ to reduce low-frequency drift and high-frequency respiratory and cardiac noise (Biswal et al., 1995). To reduce the effect of physiological artifacts, we removed effects of the following nuisance covariates: global mean signal, the six motion parameters, signal from cerebrospinal fluid and white matter (Fox et al., 2005; Kelly et al., 2008). We conducted an exploratory analysis by means of DPARSF computing functional connectivity maps with a seed region consisting of bilateral hippocampus in MNI space taken from the AAL atlas (Tzourio-Mazoyer et al., 2002).

The resulting whole-brain functional connectivity maps were compared between EFP and LFP with a significance threshold of $P<0.001$ and a cluster-size correction by means of Monte Carlo simulation. Significant effects were reported when the volume of the cluster was greater than the Monte Carlo simulation determined minimum cluster size on the whole brain volume ( $>25$ voxels), above which the probability of type I error was below 0.05 (AlphaSim, Ward, 2000).

\section{Results}

\subsection{Hormonal and behavioral data}

The mean hormonal and BDNF values and their standard errors in each cycle phase are shown in Fig. 1. For visual display of the error bars, between-subject variance was removed before plotting following 

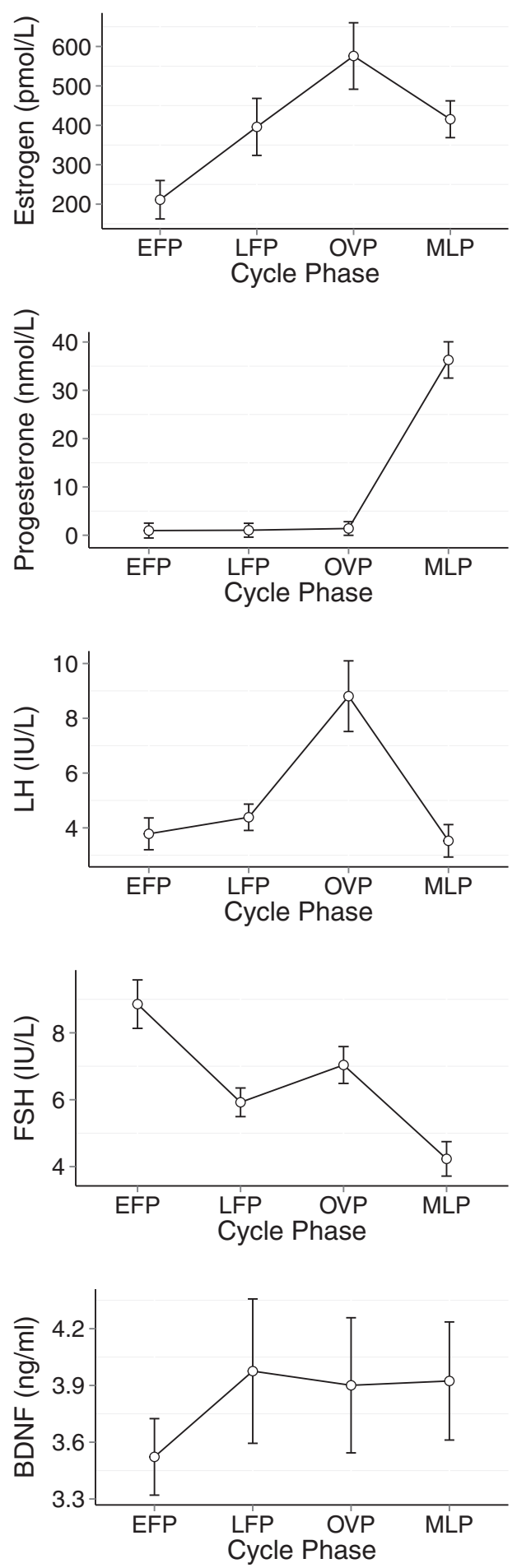

Fig. 1. Estrogen, progesterone, LH, FSH and BDNF levels on the four measurement occasions. Error bars represent standard error of the mean (SEM) at each time point after removing between-person variability (Morey, 2008).

Morey (2008). Estrogen levels were significantly higher during LFP, OVP and MLP than during EFP, and did not significantly differ between each of the three phases (LFP $>$ EFP $T(\mathrm{df}=16)=2.14, P=0.049$; OVP $>$ EFP $T(\mathrm{df}=15)=3.60, P<0.001 ; \operatorname{MLP}>\operatorname{EFP} T(\mathrm{df}=15)=2.54, P=0.023)$. Progesterone was significantly higher during the MLP than during all three other phases and did not differ between each of the three nonluteal phases $($ MLP $>$ EFP $T(\mathrm{df}=17)=7.72 ; \operatorname{MLP}>\operatorname{LFP} T(\mathrm{df}=$
$18)=7.57 ; \mathrm{MLP}>\mathrm{OVP} T(\mathrm{df}=17)=7.63$, all $P<0.001)$. The mean FSH and LH values followed the expected pattern, with peak LH levels at ovulation and elevated FSH levels during menses (EFP) and ovulation $(\mathrm{LH}:$ OVP $>$ EFP $T(\mathrm{df}=16)=3.44 P=0.003 ;$ OVP $>$ LFP $T(\mathrm{df}=15)=$ $3.49 P=0.003 ;$ OVP $>$ MLP $T(\mathrm{df}=16)=4.19 P=<0.001 ; \mathrm{FSH}$ : $\mathrm{OVP}>\operatorname{LFP} T(\mathrm{df}=13)=2.28 P=0.04 ; \mathrm{OVP}>\operatorname{MLP} T(\mathrm{df}=14)=4.22$ $P<0.001 ; \mathrm{EFP}>\operatorname{MLP} T(\mathrm{df}=19)=4.49 P<0.001)$. The hormonal levels and the expected ranges (based on Stricker et al., 2006) within each cycle phase are shown in Table 2 . The hormonal variation across the cycle corresponds to the expected range. Only two of the sixteen values fall outside the expected ranges in the respective cycle phases described by Stricker and colleagues (namely FSH at EFP and LH at OVP). We attribute these deviances to the different sampling protocol that was used in the study by Stricker, in which LH was assessed on a daily basis, making it possible to exactly determine the day of ovulation. Our ovulatory measurements were scheduled within a range of a few days surrounding ovulation, which explains the lower LH levels we observed during that phase.

Detailed descriptive results for the cognitive tasks in each cycle phase can be found in the Supplementary Table 1. Performance did not differ reliably as a function of menstrual cycle phase for any of the cognitive tasks $(P>0.006$, Bonferroni corrected $)$. Furthermore, no significant correlation between estrogen and progesterone concentration and any cognitive test performance across the four time points was observed $(P>0.006)$.

\subsection{Structural MRI}

A whole-brain VBM comparison revealed three clusters showing higher GM in the LFP compared to EFP $(P<0.0005$ uncorrected, cluster size non-stationary smoothness corrected with $\mathrm{k}>25$ voxels) Two clusters were located within the posterior hippocampi, the cluster on the right hemisphere included also thalamic regions (MNI peak coordinate left hippocampus: $-27,-38,6$; right hippocampus extending into thalamus: $14,-24$, 3; Fig. 2; extracted GM probability across the four measurement occasions is shown in Fig. 3). The third cluster was located within the thalamus (MNI $-3,-3,2)$. GM probability for each participant in the posterior hippocampi correlated negatively with the MD values (extracted from the DTI data) of the same region at both measurement occasions (EFP: $R=-53$; LFP: $R=-54$; both $P<0.05$; one subject was excluded because of outlier values on MD and GM scores). No regions were found to have higher GM volume in the EFP compared to the LFP. The left hippocampus/parahippocampal gyrus (MNI: $-36,-29,-14$ ) showed increased volume in the LFP compared to the MLP. None of the other phase contrasts revealed clusters within or nearby the hippocampus. The clusters emerging in the other contrasts can be found in Supplementary Table 2.

The VBM whole-brain one-sample t-test of within-subject correlations between estrogen values and GM density across the four occasions revealed higher GM volume in the left parahippocampal gyrus associated with higher estrogen levels (MNI: $-26,-42,-3 ; P<0.001$, cluster size $\mathrm{k}>5$, corrected for non-stationary smoothness). Two other regions were found to correlate positively with estrogen, located within left middle frontal gyrus (MNI: $-47,9,32$ ) and right cerebellum (MNI: $29,-69,-24)$. No region was found to correlate negatively with estrogen values across the four occasions.

To verify the VBM results we also extracted FreeSurfer hippocampus segmentations, which showed a significant increase in the hippocampal volume (right hippocampus + left hippocampus / 2) from EFP to LFP $(T(\mathrm{df}=20)=2.22, P=0.038$, Fig. 4$)$. At LFP, hippocampal volumes correlated positively with BDNF concentrations derived from the blood assays ( $R=0.43 ; P=0.049$ ).

The water content measure in the hippocampal regions showing significant GM changes between EFP and LFP revealed no differences in water content between the respective phases. 
Table 2

Hormonal assays and expected levels (Stricker et al., 2006).

\begin{tabular}{|c|c|c|c|c|}
\hline & Estrogen (pmol/L) & Progesterone (nmol/L) & $\mathrm{LH}(\mathrm{IU} / \mathrm{L})$ & FSH (IU/L) \\
\hline EFP & $211.201( \pm 88.70)$ & $0.965( \pm 0.49)$ & $3.782( \pm 1.94)$ & $8.858( \pm 4.83)$ \\
\hline Expected range & [77.99-266.08] & {$[0.32-1.91]$} & {$[2.01-7.80]$} & {$[3.91-8.76]$} \\
\hline LFP & $395.990( \pm 326.53)$ & $1.038( \pm 0.59)$ & $4.386( \pm 2.56)$ & $5.923( \pm 2.59)$ \\
\hline Expected range & [195.43-1146.91] & {$[0.32-1.59]$} & [3.24-14.68] & [2.97-6.99] \\
\hline OVP & $575.850( \pm 432.16)$ & $1.394( \pm 0.77)$ & $8.807( \pm 6.26)$ & $7.039( \pm 2.45)$ \\
\hline Expected range & [482.00-1425.39] & [1.24-4.13] & {$[21.16-73.63]$} & {$[5.30-15.07]$} \\
\hline MLP & $415.386( \pm 323.34)$ & $36.287( \pm 19.96)$ & $3.527(2.85)$ & $4.232(1.84)$ \\
\hline Expected range & [275.95-761.67] & [21.21-54.82] & [1.05-9.73] & {$[1.48-5.31]$} \\
\hline
\end{tabular}

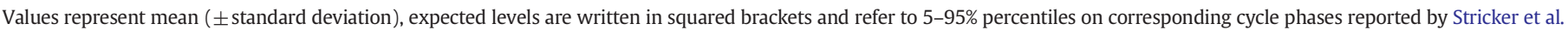
(2006); EFP = early follicular phase, LFP = late follicular phase, OVP = ovulatory phase, MLP = mid-luteal phase.

\subsection{Functional MRI}

To investigate cycle-dependent changes in brain regions that are functionally associated with both hippocampi we computed functional connectivity of a hippocampal ROI taken from the automatic anatomical labeling (AAL) atlas. The resulting connectivity maps were contrasted across cycle phases with a $P$-value of $<0.001$ (Monte-Carlo corrected). We found higher functional connectivity of the left and right hippocampus and the bilateral superior parietal lobe (MNI: $42,-54,54 ;-42$, -51, 60; Fig. 5) during LFP compared to EFP. Similar regions of higher connectivity were found when comparing LFP to MLP (MNI: -42 , $-48,57 ; 36,-60,54)$. Post-hoc analyses within the parietal clusters showed that functional connectivity reached a peak during LFP.

\section{Discussion}

In the present study, we found that the gray matter (GM) volume of both hippocampi increases from the early to the late follicular phase of the human menstrual cycle. Higher GM volumes were associated with lower mean diffusivity (MD) in the same region. Furthermore no water content changes were detected in the hippocampus. The functional connectivity of left and right hippocampus with bilateral superior parietal lobule was increased during the late follicular phase as well. The observed hippocampal variation across all cycle phases is in line with magnifying estrogen effects, which are most pronounced when not accompanied by high progesterone levels. These results offer a number of interesting new insights into cycle-related rapid human brain plasticity.

By including a measurement occasion during the late follicular phase of the cycle, in which estrogen is increased whereas all other gonadal hormones are relatively low, we could specifically compare a high with a low estrogen phase. We were able to validate the assessment

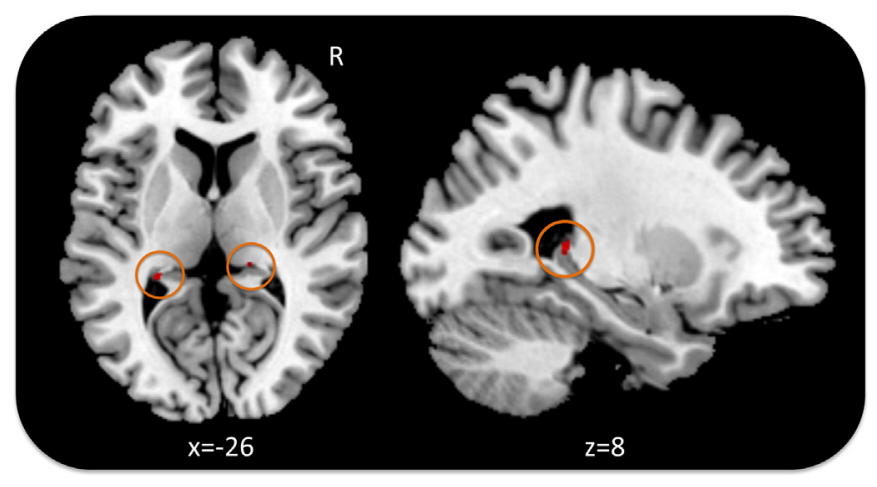

Fig. 2. Increase in gray matter hippocampal volume from the early follicular phase (EFP) to the late follicular phase (LFP). Results show greater gray matter volume in the posterior part of both hippocampi during LFP relative to EFP. $P<0.0005, \mathrm{k}>25$ voxels, corrected for nonstationary smoothness. of cycle phases by hormonal assays based on blood samples. The differences between high and low estrogen follicular phases revealed structural changes in the hippocampus that are in line with previous findings (Protopopescu et al., 2008). In addition to whole-brain VBM results, we also observed cycle-dependent hippocampal volume changes when using a volumetric subcortical measure derived from FreeSurfer. The main finding from a VBM whole-brain comparison was strengthened by evidence from the other measures and imaging modalities. We recommend replicating these additional analyses (as the DTI and fMRI results) in future studies specifically addressing each modality in order to control for alpha bias cumulation. The only other region showing GM volume increase from early to late follicular phase was the thalamus. Thalamic volume and glucose metabolism change across the human menstrual cycle were also reported by previous neuroimaging studies (Protopopescu et al., 2008; Reiman et al., 1996; Tu et al., 2013), and might be negatively related to menstrual pain (Tu et al., 2013).

Research on hippocampal brain changes during the menstrual cycle needs to address two important questions: First, how do hormonal variations during different cycle phases influence hippocampal volume, and second, which underlying neural changes contribute to the reported volume increases? Most previous studies measured brain structure or function at only two points in time during the menstrual cycle, one generally located during the early or middle follicular phase, and the other during the early, middle, or late luteal phase. To get more information about the hippocampal volume changes over the full menstrual cycle, we scanned the women on four points in time of their menstrual cycle, namely, the early follicular phase (EFP), the late follicular phase (LFP), ovulation (OVP), and the luteal phase (MLP). Significant left and right hippocampal differences were only observed in the LFP versus EFP contrast, by means of whole-brain comparison. If this volume

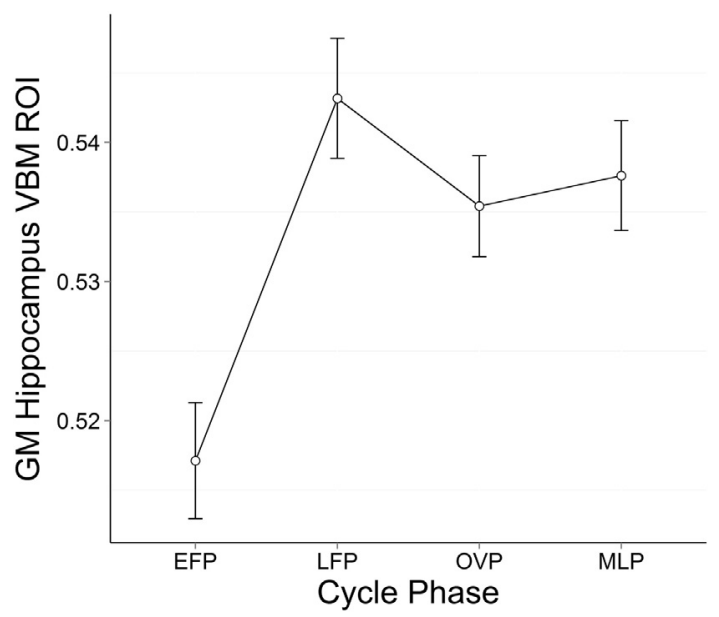

Fig. 3. GM probability in the posterior hippocampus (ROI data extracted from VBM LFP $>$ EFP contrast) across the four measurement occasions. Error bars represent standard error of the mean (SEM) at each time point after removing between-person variability (Morey, 2008). 


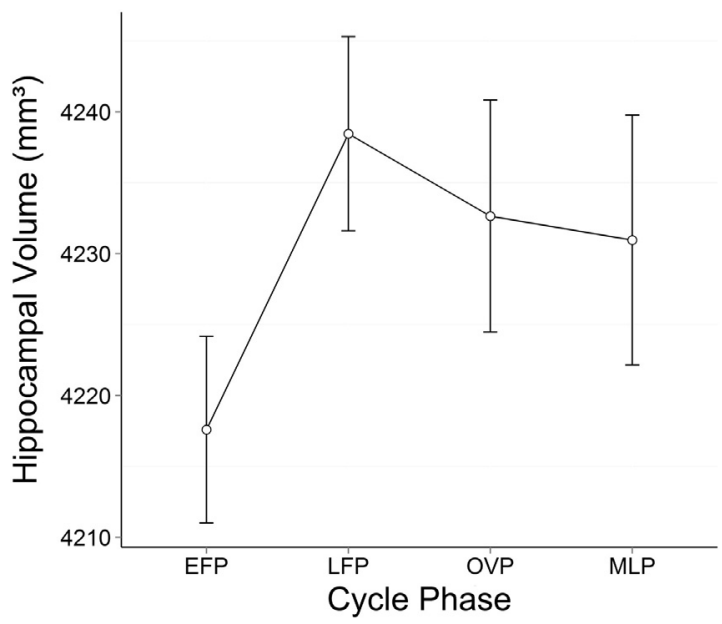

Fig. 4. FreeSurfer volume of the mean right and left hippocampi across the four measurement occasions. Error bars represent standard error of the mean (SEM) at each time point after removing between-person variability (Morey, 2008).

increase is related to the estrogen increase between the phases, the volume should remain constantly high during the high estrogen ovulatory and luteal phase and have its minimum during the early follicular phase. This assumption was confirmed in the VBM as well as in FreeSurfer volumetric measures. Nevertheless, the LFP was the only phase showing significant hippocampal GM increases in comparison to the EFP. This indicates that the effects of elevated estrogen might be influenced by other gonadal hormones, such as high progesterone levels. This would be in line with results from animal studies showing that interaction effects between estrogen and progesterone can lead to an attenuation and even inversion of estrogen-induced structural brain changes (Woolley and McEwen, 1993). Elevated progesterone levels cause a decrease in hippocampal spine density in animals and are responsible for the volume decline from proestrus to estrus (Woolley and McEwen, 1993). Our results resemble these mechanisms. The left hippocampus/parahippocampal gyrus showed significantly higher volume in the LFP compared to MLP. While estrogen levels were comparable between the two phases, high progesterone levels during MLP could explain the smaller volume. Taken together, the present results strengthen the hypothesis that estrogen influences hippocampal volumes in humans in comparable ways as it does in animals. Therefore, the results confirmed that the high estrogen LFP (and to a lesser extent the MLP) are good contrasts to the low estrogen EFP when assessing hippocampal plasticity. The more extensive design in the present study controlled, that hippocampal change follows the pattern that was assumed in previous studies.

The question of the underlying neuronal mechanisms through which hormones influence brain structure in humans still remains to

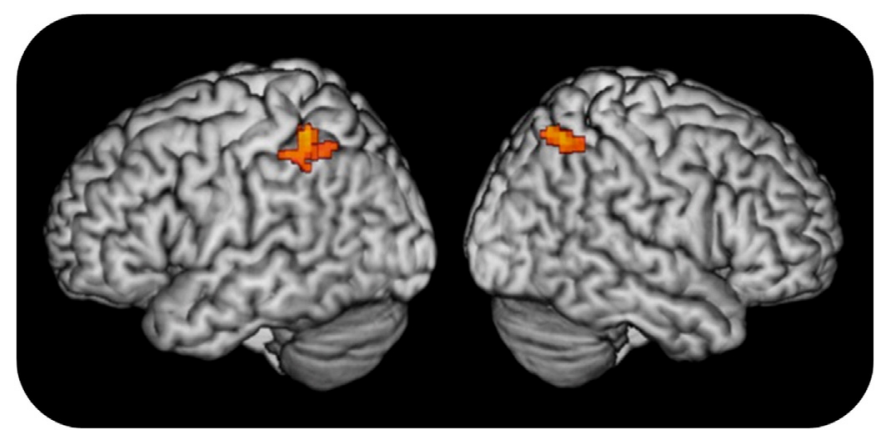

Fig. 5. Increase in functional connectivity of the left and right hippocampus with the bilateral superior parietal lobe from the early follicular phase (EFP) to the late follicular phase (LFP). $P<0.001$, Monte Carlo corrected. be answered. Our results provide first evidence about possible mechanisms. Across subjects, gray matter density in the posterior hippocampus correlated strongly with the MD values at the early and late follicular phase. Lower MD values in GM indicate that water molecules are hindered more in their natural diffusion in these GM regions, which may point to tighter density of GM (Mackey et al., 2012), for example caused by changes in glia cells (Zatorre et al., 2012). By including a water content measure, we were able to ascertain that the volume increase within the hippocampus was not based on water content changes. In conjunction, the present pattern of results suggests that the volume increase in the hippocampus during the LFP is due to structural changes at the micro-cellular level and not only a side-effect of hormone-related water-shifts in brain (Zuckerman et al., 1950). Possible mechanisms for these micro-cellular changes include synaptogenesis, angiogenesis and hypertrophy (Hagemann et al., 2011; Zatorre et al., 2012). Estrogen is known to affect cell proliferation as well as cell survival in the hippocampus in adult female animals (Galea et al., 2006; Pawluski et al., 2009). Hippocampal estrogen receptors have been proposed as one pathway of estrogen effects on neural plasticity (Spencer et al., 2008). These receptors are also present in the human hippocampi (Österlund and Hurd, 2001; Tohgi et al., 1995).

In line with cycle-dependent structural alterations, we also observed differences in functional connectivity when the hippocampus was used as a seed region. Estrogen effects on functional brain organization have been previously demonstrated in studies on interhemispheric inhibition (Weis et al., 2008, 2011). In the present study, the connectivity between the hippocampus and the superior parietal lobe was significantly strengthened during the LFP. Previous fMRI studies showed functional connectivity between these brain regions during spatial tasks, for instance, during flexible retrieval of spatial information in navigation (Zhang and Ekstrom, 2012). The superior parietal lobe itself also seems to be sensitive to hormonal variation across the cycle. Schöning et al. (2007) found a correlation between the activation of the superior parietal lobe during a mental rotation task and the serum estradiol concentration (Schöning et al., 2007). Interpreting the hippocampalparietal connectivity in connection to spatial processing seems plausible given that the hippocampus itself, especially the posterior part, is also involved in spatial processing (Kühn and Gallinat, 2014). Therefore, our finding of higher connectivity between the regions during highestrogen phases of the menstrual cycle is in line with previous behavioral results documenting cycle-dependent differences in performance on spatial tasks (McCormick and Teillon, 2001; Schöning et al., 2007; Silverman and Phillips, 1993). Future studies need to clarify why high estrogen phases of the human menstrual cycle are associated with an increase in the functional connectivity of the hippocampus, and a decrease in performance on spatial tasks, such as mental rotation (Silverman and Phillips, 1993). Referring to animal data, different authors suggested reasons why increased hippocampal neurogenesis or spine density could be related to decreased cognitive performance. Worse hippocampal signal to noise ratio due to more highly excitable new neurons is one example (Pawluski et al., 2009; Woolley, 1998).

Contrary to our expectations, we did not observe any reliable cyclerelated variations in cognitive performance. As a consequence, we were not able to observe any reliable cycle-related covariation between cognitive performance and hippocampal volume. We attribute this lack of findings to two limitations of our study. First, the sample size of the present study was rather small, and may have lacked the statistical power to detect cycle-related within-person fluctuations in performance. Second, we only assessed a limited number of cognitive tasks from a broad range of cognitive abilities, such as working and episodic memory, perceptual speed and spatial cognition. We selected tasks from a battery that has been shown to be applicable in a multiplemeasurements design (Schmiedek et al., 2010). These tasks did not include tests for specific spatial abilities, such as mental rotation, which previously have been associated with menstrual cycle-related performance differences. For some of the tasks, retest and ceiling effects 
might have further limited the potential to find differences across cycle phases.

It is worth noting that the results of previous studies measuring cognition during different cycle phases are not consistent. Some studies observed performance differences over the menstrual cycle in tasks measuring spatial and verbal abilities as well as memory (Hampson, 1990; Hausmann et al., 2000; Maki et al., 2002; Rosenberg and Park, 2002), domains that are known to be related to hippocampal function (Kühn and Gallinat, 2014). However, some studies report no cyclerelated variations in performance on verbal and visuospatial tests (e.g., Epting and Overman, 1998; Gordon and Lee, 1993). In a recent study, Mihalj et al. (2014) did not find any reliable cycle-phaserelated differences on a number of cognitive tasks in a controlled sample of young medicine students, similar to the present sample. In this study, no specific tasks testing navigational skills were implemented as well. Given the inconsistent evidence in the literature so far, we strongly suggest that future studies should assess cycle-related effects on a range of different spatial abilities with highly reliable and change-sensitive tasks and samples of adequate size.

As a last point we would like to discuss a general methodological issue that is of importance when targeting the structural plasticity of the brain. Our data strongly suggest that the menstrual cycle should be considered when planning longitudinally as well as cross-sectional MR-studies with women. Training studies are one prominent example of experimental designs, whose results could be substantially biased by the particular cycle phase of the participating women. When conducting those studies, researchers should pay careful attention to the cycle phases of their female subjects, in order to not confound the results with hormone related plasticity.

In summary, the present study indicates that volume and functional connectivity of the human female hippocampus vary as a function of the menstrual cycle. It is likely that these variations are connected to cyclic variations in hormonal levels, especially estrogen, as estrogen was selectively elevated during the late follicular phase. The pattern of hippocampal plasticity across the full cycle parallels findings from animal studies and suggests comparable hormone-brain interactions. The present findings have important implications for neuroscience research with young adult women. In particular, they suggest that a reliable portion of observed structural and functional between-person variability, especially in young, age-homogenous samples, may reflect cyclerelated within-person fluctuations instead of stable between-person differences. The magnitude and consequences of this confound merit further study. On a more general level, the present results contribute to the growing knowledge about short-term structural brain plasticity in the human brain (Lövdén et al., 2013).

Supplementary data to this article can be found online at http://dx. doi.org/10.1016/j.neuroimage.2015.06.012.

\section{Conflict of interest}

The authors declare that there is no conflict of interest.

\section{Acknowledgments}

We are grateful for the assistance of Ludmila Müller and the MRI team at the MPI Berlin consisting of Sonali Beckmann, Nils Bodammer, Thomas Feg, Sebastian Schröder, Nadine Taube, and we thank our student assistants for their help with data collection and preparation.

\section{References}

Andersson, J.L.R., Jenkinson, M., Smith, S., 2007a. Non-linear Optimisation FMRIB Technial Report TR07JA1 (June).

Andersson, J.L.R., Jenkinson, M., Smith, S., 2007b. Non-linear Registration Aka Spatia Normalisation FMRIB Technial Report TR07JA2 (June).
Avants, B.B., Epstein, C.L., Grossman, M., Gee, J.C., 2008. Symmetric diffeomorphic image registration with cross-correlation: evaluating automated labeling of elderly and neurodegenerative brain. Med. Image Anal. 12 (1), 26-41.

Avants, B., Yushkevich, P., Pluta, J., 2010. The optimal template effect in hippocampus studies of diseased populations. Neurolmage 49 (3), 1-23.

Avants, B., Tustison, N., Song, G., Cook, P., 2011. A reproducible evaluation of ANTs similarity metric performance in brain image registration. Neurolmage 54 (3), 2033-2044.

Bimonte, H.A., Denenberg, V.H., 1999. Estradiol facilitates performance as working memory load increases. Psychoneuroendocrinology 24 (2), 161-173.

Biswal, B., Yetkin, F.Z., Haughton, V.M., Hyde, J.S., 1995. Functional connectivity in the motor cortex of resting. Magn. Reson. Med. 34 (1995), 537-541.

Chao-Gan, Y., Yu-Feng, Z., 2010. DPARSF: a MATLAB toolbox for "pipeline" data analysis of resting-state fMRI. Front. Syst. Neurosci. 4 (May), 13.

Chesler, E.J., Juraska, J.M., 2000. Acute administration of estrogen and progesterone impairs the acquisition of the spatial morris water maze in ovariectomized rats. Horm. Behav. 38 (4), 234-242.

Czoty, P.W., Riddick, N.V., Gage, H.D., Sandridge, M., Nader, S.H., Garg, S., Nader, M.a., 2009. Effect of menstrual cycle phase on dopamine D2 receptor availability in female cynomolgus monkeys. Neuropsychopharmacology 34 (3), 548-554.

De Bondt, T., Jacquemyn, Y., Van Hecke, W., Sijbers, J., Sunaert, S., Parizel, P.M., 2013. Regional gray matter volume differences and sex-hormone correlations as a function of menstrual cycle phase and hormonal contraceptives use. Brain Res. 1530, 22-31.

Desmond, N.L., Levy, W.B., 1997. Ovarian steroidal control of connectivity in the female hippocampus: an overview of recent experimental findings and speculations on its functional consequences. Hippocampus 7 (2), 239-245.

Epting, L., Overman, W., 1998. Sex-sensitive tasks in men and women: a search for performance fluctuations across the menstrual cycle. Behav. Neurosci. 112 (6), 1304-1317.

Fader, A.J., Hendricson, A.W., Dohanich, G.P., 1998. Estrogen improves performance of reinforced T-maze alternation and prevents the amnestic effects of scopolamine administered systemically or intrahippocampally. Neurobiol. Learn. Mem. 69 (3), 225-240.

Fox, M.D., Snyder, A.Z., Vincent, J.L., Corbetta, M., Van Essen, D.C., Raichle, M.E., 2005. The human brain is intrinsically organized into dynamic, anticorrelated functional networks. Proc. Natl. Acad. Sci. U. S. A. 102 (27), 9673-9678.

Galea, L.A., Kavaliers, M., 1995. Gonadal hormone levels and spatial learning performance in the Morris water maze in male and female meadow voles, Microtus pennsylvanicus. Horm. Behav. 29, 106-125.

Galea, L.A., Spritzer, M.D., Barker, J.M., Pawluski, J.L., 2006. Gonadal hormone modulation of hippocampal neurogenesis in the adult. Hippocampus 16 (3), 225-232.

Gibbs, R.B., 2000. Long-term treatment with estrogen and progesterone enhances acquisition of a spatial memory task by ovariectomized aged rats. Neurobiol. Aging 21 (1), 107-116.

Gordon, H.W., Lee, P.A., 1993. No difference in cognitive performance between phases of the menstrual cycle. Psychoneuroendocrinology 18 (7), 521-531.

Guilford, J.P., Zimmerman, W.S., 1948. The Guilford-Zimmerman aptitude survey. J. Appl. Psychol. 32 (1), 24-34.

Hagemann, G., Ugur, T., Schleussner, E., Mentzel, H.-J., Fitzek, C., Witte, O.W., Gaser, C., 2011. Changes in brain size during the menstrual cycle. PLoS One 6 (2), e14655.

Hampson, E., 1990. Variations in sex-related cognitive abilities across the menstrual cycle. Brain Cogn. 14 (1), 26-43.

Hausmann, M., Slabbekoorn, D., Van Goozen, S.H., Cohen-Kettenis, P.T., Güntürkün, O., 2000. Sex hormones affect spatial abilities during the menstrual cycle. Behav. Neurosci. 114 (6), 1245-1250.

Hayasaka, S., Nichols, T.E., 2004. Combining voxel intensity and cluster extent with permutation test framework. Neurolmage 23 (1), 54-63.

Hussain, D., Waqqas, S., Wayne, B., 2014. Estrogen and memory system bias in females across the lifespan. Transl. Neurosci. 5 (1), 35-50.

Kelly, A.M.C., Uddin, L.Q., Biswal, B.B., Castellanos, F.X., Milham, M.P., 2008. Competition between functional brain networks mediates behavioral variability. NeuroImage 39 (1), 527-537.

Klein, A., Ghosh, S., Avants, B., Yeo, B., 2010. Evaluation of volume-based and surfacebased brain image registration methods. Neurolmage 51 (1), 214-220.

Korol, D.L., Kolo, L.L., 2002. Estrogen-induced changes in place and response learning in young adult female rats. Behav. Neurosci. 116 (3), 411-420.

Korol, D.L., Malin, E.L., Borden, K. a, Busby, R. a, Couper-Leo, J., 2004. Shifts in preferred learning strategy across the estrous cycle in female rats. Horm. Behav. 45 (5), 330-338.

Kühn, S., Gallinat, J., 2014. Segregating cognitive functions within hippocampal formation: a quantitative meta-analysis on spatial navigation and episodic memory. Hum. Brain Mapp. 35 (4), 1129-1142.

Lacreuse, A., Verreault, M., Herndon, J.G., 2001. Fluctuations in spatial recognition memory across the menstrual cycle in female rhesus monkeys. Psychoneuroendocrinology 26 (6), 623-639.

Lövdén, M., Wenger, E., Mårtensson, J., Lindenberger, U., Bäckman, L., 2013. Structural brain plasticity in adult learning and development. Neurosci. Biobehav. Rev. 37 (9 Pt B), 2296-2310

Mackey, A.P., Whitaker, K.J., Bunge, S.A., 2012. Experience-dependent plasticity in white matter microstructure: reasoning training alters structural connectivity. Front. Neuroanat. 6 (August), 32.

Maki, P.M., Rich, J.B., Rosenbaum, R.S., 2002. Implicit memory varies across the menstrual cycle: estrogen effects in young women. Neuropsychologia 40 (5), 518-529.

McCormick, C.M., Teillon, S.M., 2001. Menstrual cycle variation in spatial ability: relation to salivary cortisol levels. Horm. Behav. 39 (1), 29-38.

Mihalj, M., Drenjančević, I., Včev, A., Šumanovac, A., Čavka, A., Gmajnić, R., 2014. Basic cognitive functions across the menstrual cycle in a controlled female cohort. Med. Glas. (Zenica) 11 (1), 177-185. 
Morey, R.D., 2008. Confidence intervals from normalized data: a correction to Cousineau (2005). Reason 4 (2), 61-64.

Morey, R.A., Petty, C.M., Xu, Y., Hayes, J.P., Wagner, H.R., Lewis, D.V., McCarthy, G., 2009. A comparison of automated segmentation and manual tracing for quantifying hippocampal and amygdala volumes. NeuroImage 45 (3), 855-866.

Neeb, H., Ermer, V., Stocker, T., Shah, N.J., 2008. Fast quantitative mapping of absolute water content with full brain coverage. NeuroImage 42 (3), 1094-1109.

O'Neal, M., Means, L., Poole, M., Hamm, R., 1996. Estrogen affects performance of ovariectomized rats in a two-choice water-escape working memory task. Psychoneuroendocrinology 21 (1), 51-65.

Olmos, G., Naftolin, F., Perez, J., Tranque, P.A., Garcia-Segura, L.M., 1989. Synaptic remodeling in the rat arcuate nucleus during the estrous cycle. Neuroscience 32 (3), 663-667.

Ossewaarde, L., van Wingen, G.A., Rijpkema, M., Bäckström, T., Hermans, E.J., Fernández, G., 2011. Menstrual cycle-related changes in amygdala morphology are associated with changes in stress sensitivity. Hum. Brain Mapp. 000 (September).

Österlund, M., Hurd, Y., 2001. Estrogen receptors in the human forebrain and the relation to neuropsychiatric disorders. Prog. Neurobiol. 64, 251-267.

Pawluski, J.L., Brummelte, S., Barha, C.K., Crozier, T.M., Galea, L.A.M., 2009. Effects of steroid hormones on neurogenesis in the hippocampus of the adult female rodent during the estrous cycle, pregnancy, lactation and aging. Front. Neuroendocrinol. 30 (3), 343-357.

Pletzer, B., Kronbichler, M., Aichhorn, M., Bergmann, J., Ladurner, G., Kerschbaum, H.H., 2010. Menstrual cycle and hormonal contraceptive use modulate human brain structure. Brain Res. 1348, 55-62.

Protopopescu, X., Butler, T., Pan, H., Root, J., Altemus, M., Polanecsky, M., Stern, E., 2008. Hippocampal structural changes across the menstrual cycle. Hippocampus 18 (10), 985-988.

Qiu, L.R., Germann, J., Spring, S., Alm, C., Vousden, D. a, Palmert, M.R., Lerch, J.P., 2013. Hippocampal volumes differ across the mouse estrous cycle, can change within 24 hours, and associate with cognitive strategies. Neurolmage 83C, 593-598.

Reiman, E.M., Armstrong, S.M., Matt, K.S., Mattox, J.H., 1996. The application of positron emission tomography to the study of the normal menstrual cycle. Hum. Reprod. 11 (12), 2799-2805.

Rosenberg, L., Park, S., 2002. Verbal and spatial functions across the menstrual cycle in healthy young women. Psychoneuroendocrinology 27 (7), 835-841.

Schmiedek, F., Lövdén, M., Lindenberger, U., 2010. Hundred days of cognitive training enhance broad cognitive abilities in adulthood: findings from the COGITO study. Front. Aging Neurosci. 2 (July), 1-10.

Schöning, S., Engelien, A., Kugel, H., Schäfer, S., Schiffbauer, H., Zwitserlood, P., Konrad, C., 2007. Functional anatomy of visuo-spatial working memory during mental rotation is influenced by sex, menstrual cycle, and sex steroid hormones. Neuropsychologia 45 (14), 3203-3214

Silverman, I., Phillips, K., 1993. Effects of estrogen changes during the menstrual cycle on spatial performance. Ethol. Sociobiol. 270, 257-269.

Smith, S.M., 2002. Fast robust automated brain extraction. Hum. Brain Mapp. 17 (3), 143-155.

Spencer, J.L., Waters, E.M., Romeo, R.D., Wood, G.E., Milner, T. a, McEwen, B.S., 2008. Uncovering the mechanisms of estrogen effects on hippocampal function. Front Neuroendocrinol. 29 (2), 219-237.
Stackman, R.W., Blasberg, M.E., Langan, C.J., Clark, A.S., 1997. Stability of spatial working memory across the estrous cycle of Long-Evans rats. Neurobiol. Learn. Mem. 67 (2), 167-171.

Stricker, R., Eberhart, R., Chevailler, M.C., Quinn, F.a., Bischof, P., Stricker, R., 2006. Establishment of detailed reference values for luteinizing hormone, follicle stimulating hormone, estradiol, and progesterone during different phases of the menstrual cycle on the Abbott ARCHITECT ${ }^{\circledR}$ analyzer. Clin. Chem. Lab. Med. 44 (7), 883-887.

Tohgi, H., Utsugisawa, K., Yamagata, M., Yoshimura, M., 1995. Effects of age on messenge RNA expression of glucocorticoid, thyroid hormone, androgen, and estrogen receptors in postmortem human hippocampus. Brain Res. 700 (1-2), 245-253.

Tonkova, V., Arhelger, V., Schenk, J., Neeb, H., 2012. Rapid myelin water content mapping on clinical MR systems. Z. Med. Phys. 22 (2), 133-142.

Tu, C.-H., Niddam, D.M., Yeh, T.-C., Lirng, J.-F., Cheng, C.-M., Chou, C.-C., Hsieh, J.-C., 2013. Menstrual pain is associated with rapid structural alterations in the brain. Pain 154 (9), 1718-1724.

Tzourio-Mazoyer, N., Landeau, B., Papathanassiou, D., Crivello, F., Etard, O., Delcroix, N. Joliot, M., 2002. Automated anatomical labeling of activations in SPM using a macroscopic anatomical parcellation of the MNI MRI single-subject brain. NeuroImage 15 (1), 273-289.

Ward, B., 2000. Simultaneous Inference for fMRI Data. AFNI 3dDeconvolve Documentation, Medical (... Retrieved from). http://afni-dev.nimh.nih.gov/pub/dist/doc/ manual/AlphaSim.ps.

Weis, S., Hausmann, M., Stoffers, B., Vohn, R., Kellermann, T., Sturm, W., 2008. Estradiol modulates functional brain organization during the menstrual cycle: an analysis of interhemispheric inhibition. J. Neurosci. 28 (50), 13401-13410.

Weis, S., Hausmann, M., Stoffers, B., Sturm, W., 2011. Dynamic changes in functional cerebral connectivity of spatial cognition during the menstrual cycle. Hum. Brain Mapp. 32 (10), 1544-1556.

Woolley, C.S., 1998. Estrogen-mediated structural and functional synaptic plasticity in the female rat hippocampus. Horm. Behav. 34 (2), 140-148.

Woolley, C.S., McEwen, B.S., 1990. Naturally occurring fluctuation in dendritic hippocampal pyramidal neurons spine density on adult. J. Neurosci. 10 (December), 4035-4039.

Woolley, C.S., McEwen, B.S., 1993. Roles of estradiol and progesterone in regulation of hippocampal dendritic spine density during the estrous cycle in the rat. J. Comp. Neurol. 336 (2), 293-306.

Woolley, C.S., Wenzel, H.J., Schwartzkroin, P.A., 1996. Estradiol increases the frequency of multiple synapse boutons in the hippocampal CA1 region of the adult female rat. J. Comp. Neurol. 373 (1), 108-117.

Woolley, C.S., Weiland, N.G., McEwen, B.S., Schwartzkroin, P.A., 1997. Estradiol increases the sensitivity of hippocampal CA1 pyramidal cells to NMDA receptor-mediated synaptic input: correlation with dendritic spine density. J. Neurosci. 17 (5), 1848-1859.

Zatorre, R.J., Fields, R.D., Johansen-Berg, H., 2012. Plasticity in gray and white: neuroimaging changes in brain structure during learning. Nat. Neurosci. 15 (4), 528-536.

Zhang, H., Ekstrom, A., 2012. Human neural systems underlying rigid and flexible forms of allocentric spatial representation. Hum. Brain Mapp. 000.

Zuckerman, S., Palmer, A., Hanson, D.A., 1950. The effect of steroid hormones on the water content of tissues. J. Endocrinol. 6 (3), 261-276. 